\section{The Impact of Early Referrals to Dietitians for Patients With Esophagogastric Cancer}

\author{
Eric J. Roeland, MD, and Richard F. Dunne, MD
}

W eight loss is a physical representation of the impact of cancer on the body and loss of control. For patients with esophagogastric cancer (EGC), loss of appetite and weight loss lead to reduced quality of life and poor clinical outcomes. In this issue of JNCCN, Dijksterhuis et al ${ }^{1}$ present data evaluating the prevalence of pretreatment cachexia, association of pretreatment cachexia with survival, and early integration of dietitians in the care of patients with EGC. This 3-year Dutch study included $>400$ patients with EGC across all stages. Half of the patients had cachexia at presentation, which was associated with decreased survival. In our opinion, the key finding of this study is that 3-month median weight loss was lower for the patients referred to a dietitian at diagnosis (0\% vs $2 \% ; P=.047) .{ }^{1}$ These findings suggest that early involvement of dietitians may slow or stabilize weight loss in the care of patients with EGC and highlight 3 key take-away points for practicing oncologists: (1) clarify the difference between weight loss and cachexia, (2) identify precachexia and (3) refer early to dietitians.

\section{Clarify the Difference Between Weight Loss and Cachexia}

In this study, the investigators refer to "cachexia" and "weight loss" interchangeably, which is common across clinical practice. However, as clinicians, we must be clear about these terms, especially when talking to patients and caregivers. Words matter. In fact, most patients and caregivers are unaware of the term "cachexia." The optimal time to introduce cachexia is when patients and caregivers express concerns and frustrations about loss of appetite and weight loss. Weight loss is a component of cancer cachexia but does not define it. Instead, we introduce and describe cancer cachexia as a multifactorial syndrome characterized by loss of appetite, weight, and skeletal muscle, leading to fatigue, functional impairment, increased treatment-related toxicity, poor quality of life, and reduced survival. ${ }^{2}$ Oftentimes, labeling the patient experience as cachexia rather than focusing solely on weight loss helps mitigate mounting tensions between patients and their caregivers regarding food. Although this may seem like a trivial point, in our experience, explaining the differences between weight loss and cachexia minimizes blame and guilt. When the issue of weight loss arises, do not miss a key opportunity to educate.

\section{Identify Precachexia}

Most clinicians define cachexia as emaciation and frailty in a patient approaching death, and might be surprised to learn that there are 3 described stages of cancer cachexia: precachexia, cachexia, and refractory cachexia. ${ }^{3}$ In precachexia, patients experience only minimal weight loss (ie, $2 \%-5 \%$ ), with early clinical and metabolic changes (eg, anorexia, insulin resistance, inflammation, and hypogonadism) predictive of future weight loss and poor clinical outcomes. In contrast, the onset of cachexia is characterized in three ways: (1) weight loss $>5 \%$ over the preceding 6 months; (2) body mass index $<20 \mathrm{~kg} / \mathrm{m}^{2}$ with ongoing $>2 \%$ weight loss; or (3) depletion of muscle mass and $>2 \%$ weight loss. Finally, refractory cachexia is a

\section{See page 144 for related article.}

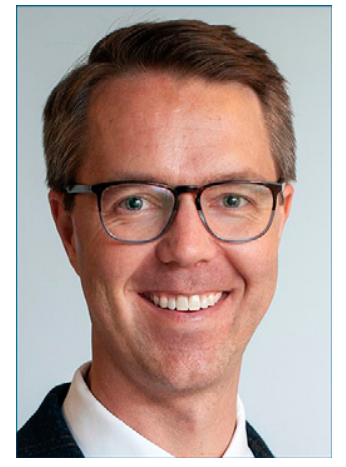

ERIC J. ROELAND, MD

Eric J. Roeland, MD, is a dual-trained and board-certified physician in palliative care and medical oncologist working as an Assistant Professor at the Massachusetts General Hospital Cancer Center. His oncology clinical practice focuses on the care of patients with gastrointestinal cancer, while his palliative care practice includes patients with all types of cancer. His clinical research focuses on identifying and evaluating novel approaches to minimize suffering and maximize the quality of life for patients with cancer.

doi: $10.6004 /$ jnccn.2021.7006

The ideas and viewpoints expressed in this commentary are those of the author and do not necessarily represent any policy, position, or program of NCCN 


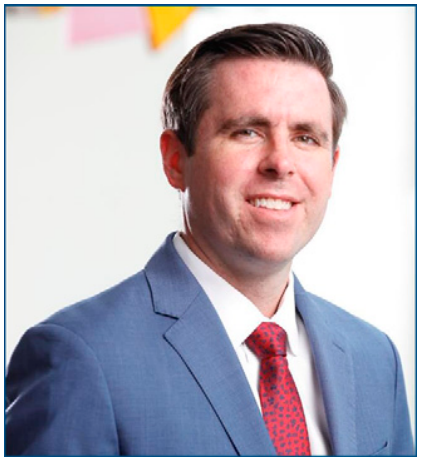

RICHARD F. DUNNE, MD

Richard F. Dunne, MD, is an Assistant Professor of medicine and medical oncologist with expertise in gastrointestinal tract cancers at the Wilmot Cancer Institute and University of Rochester Medical Center. He currently is an NIH Clinical and Translational Science Award KL2 scholar studying exercise and nutritional interventions to treat cancer cachexia. clinically resistant catabolic state characterized by limited performance status, progressive cancer, and a life expectancy $<3$ months. In our experience, when colleagues refer to cachexia they are usually referring to end-stage wasting, when medical interventions are by definition refractory. Not all patients progress through all stages of cachexia, and the risk of progression depends on multiple factors, including cancer type and stage, tumor biology, and response to cancer therapy.

Unfortunately, reliance on weight loss alone to define cachexia leads to late recognition and prevents patients from receiving important early interventions, including cancer therapy, symptom palliation, nutrition, exercise, and psychosocial support. As has likely occurred in the study by Dijksterhuis et al ${ }^{1}$, we argue that considering only weight loss and body mass index underestimates the prevalence of cancer cachexia. Weight loss measures ignore other changes, such as occult skeletal muscle loss and adipose tissue gain, which when present are useful for detecting patients at high risk for poor outcomes. ${ }^{4}$ Strategies to identify precachexia may include routine assessment of patient-reported outcomes (PROs) and body composition.

In this study, the investigators included a PRO at presentation to identify cancer cachexia. As PROs are becoming increasingly part of the standard of care in oncology practice, this may be a highly feasible and practical strategy for early identification. For example, determining thresholds of PROs may trigger consultations with dietitians, physical therapists, palliative care specialists, psychologists, and social workers.

Another strategy becoming increasingly more practical is the routine assessment of skeletal muscle on CT scans. Despite the availability of body composition data on CT scans obtained as part of usual care, oncologists primarily use CT scans to monitor tumor response to treatment. ${ }^{5}$ Although research has shown that CT-derived body composition data provide potential information to predict toxicity and poor clinical outcomes, ${ }^{6-9}$ radiologists have not routinely quantified body composition because it is considered overly burdensome. However, body composition analysis on CT scans is becoming increasingly available as artificial intelligence algorithms are being developed that are capable of performing body compartment (muscle/adipose) segmentation..$^{10,11}$ For example, fully automated algorithms exist to process thousands of $\mathrm{CT}$ images using machine learning. ${ }^{10,12}$ As a radiologic biomarker, this approach could help integrate body composition assessments into routine cancer care. Moreover, given that $\mathrm{CT}$ scans are obtained at diagnosis and used to assess treatment response, repurposing scans obtained as part of the standard of care has no cost or additional radiation exposure.

\section{Refer Early to Dietitians}

In this study, patients who met the authors' specified criteria for cachexia and were referred to a dietitian at diagnosis experienced subsequent weight stability over 3 months compared with those who were not referred. We should interpret these findings with caution given recall bias (dietitian referral was patient-reported, not abstracted from the medical chart) and lack of control for practice location or clinician, because clinicians who refer to dietitians may engage in more intensive supportive care. Unfortunately, only about half to two-thirds of patients who experienced weight loss were referred to a dietitian. This mirrors our observations that late referrals to dietitians are common when cachexia is advanced and irreversible. ${ }^{13,14}$ Several reasons exist for the underutilization of dietetic services in patients with cachexia. First, there is a systematic failure to address nutrition and cachexia-associated symptoms in oncology. For example, a qualitative research study found that clinicians miss opportunities to discuss, treat, and educate patients and caregivers regarding cachexia due to poor knowledge, a culture of avoidance, fear of causing distress, and limited time and resources. ${ }^{15}$ This missed opportunity stands in contrast to surveys of patients with cancer cachexia who report that they 
want to discuss weight loss and nutrition with their clinicians. ${ }^{16}$ Second, cachexia among patients with cancer is poorly recognized by clinicians, especially when patients are obese and have low muscle mass (ie, sarcopenic obesity), leading to increased treatment-related toxicity and poor survival. ${ }^{17,18}$

Finally, we believe the most significant explanation for underuse of dietitian expertise is an issue of access. Approximately three-quarters of institutions have dedicated dietitians working with patients with cancer, but these departments are understaffed. ${ }^{19}$ Evidence suggests a dietitian-to-patient ratio of approximately $1: 120$ is most likely to improve quality of life and nutrition outcomes; this is much different than the current ratio of 1:2,300 in most settings. ${ }^{20}$ Therefore, we believe a crucial role of practicing oncologists is to advocate for increased numbers of and access to dietitians. In fact, dietitians should perform a nutritional assessment for every patient with cancer, especially those at the highest risk for malnutrition, such as patients with EGC. This advocacy must also include developing self-sustaining business models, aside from philanthropy, to ensure consistent access to dietitians for patients with cancer.

Many oncologists perceive cancer cachexia as an eventual and unavoidable complication of progressive cancer. In fact, a central tenet in oncology is that treating the cancer is the most effective method to minimize cachexia-in other words, effective cancer treatment is optimal palliation. Although we agree that effective antineoplastic treatment may mitigate weight and muscle loss, we believe this approach should be expanded to include nutrition, symptom palliation, research, exercise, mental health, caregiver support, and education (Figure 1). To achieve this expanded model, oncologists must clarify the difference between weight loss and cachexia, identify precachexia, and refer early to dietitians. The study by Dijksterhuis et al supports this integrated model of care. Cancer cachexia, as a hypermetabolic syndrome, requires multidisciplinary collaboration. As cancer care becomes increasingly complex, our success will depend on our ability to collaborate, communicate, and advocate.

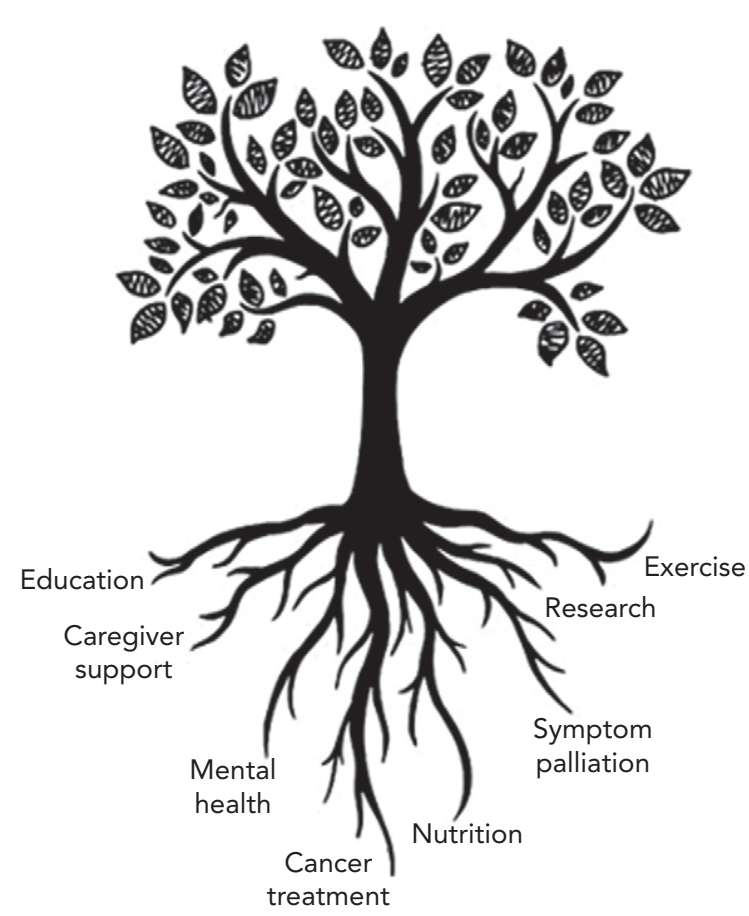

Figure 1. A comprehensive approach to the care of patients with esophagogastric cancer experiencing cachexia, including cancer treatment, nutrition, symptom palliation, research, exercise, mental health, caregiver support, and education.

Disclosures: Dr. Roeland has disclosed that he is a consultant for Mitobridge Inc., Asahi Kasei Pharmaceuticals, DRG Consulting, Napo Pharmaceuticals, American Imaging Management, Immuneering Corporation, and Prime Oncology. Additionally, he has served on recent advisory boards for Heron Pharmaceuticals, Vector Oncology, and Helsinn Pharmaceuticals. He has also served as a member on data safety monitoring boards for Oragenics, Inc, Galera Pharmaceuticals, and Enzychem Lifesciences Pharmaceutical Company. Dr. Dunne has disclosed that he is a consultant or on an advisory board for Exelixis Inc.

Funding: Dr. Dunne is supported by the University of Rochester CTSA award number KL2TR001999 from the National Center for Advancing Translational Sciences of the National Institutes of Health (RFD).

Correspondence: Eric J. Roeland, MD, Massachusetts General Hospital Cancer Center, 55 Fruit Street, Yawkey 7E, Boston, MA 02114.

Email: eroeland@mgh.harvard.edu

\section{References}

1. Dijksterhuis WPM, Latenstein AEJ, van Kleef JJ, et al. Cachexia and dietetic interventions in patients with esophagogastric cancer: a multicenter cohort study. J Natl Compr Canc Netw 2021;19:144-152.

2. Roeland EJ, Bohlke K, Baracos VE, et al. Management of cancer cachexia: ASCO guideline. J Clin Oncol 2020;38:2438-2453.

3. Fearon K, Strasser F, Anker SD, et al. Definition and classification of cancer cachexia: an international consensus. Lancet Oncol 2011;12:489-495.

4. Martin L, Senesse P, Gioulbasanis I, et al. Diagnostic criteria for the classification of cancer-associated weight loss. J Clin Oncol 2015;33: 90-99.

5. Eisenhauer EA, Therasse P, Bogaerts J, et al. New Response Evaluation Criteria in Solid Tumours: revised RECIST guideline (version 1.1). Eur J Cancer 2009;45:228-247.

6. Shen W, Punyanitya M, Wang Z, et al. Total body skeletal muscle and adipose tissue volumes: estimation from a single abdominal crosssectional image. J Appl Physiol (1985) 2004;97:2333-2338.

7. Mourtzakis M, Prado CM, Lieffers JR, et al. A practical and precise approach to quantification of body composition in cancer patients using computed tomography images acquired during routine care. Appl Physiol Nutr Metab 2008;33:997-1006.

8. Lieffers JR, Mourtzakis M, Hall KD, et al. A viscerally driven cachexia syndrome in patients with advanced colorectal cancer: contributions of organ and tumor mass to whole-body energy demands. Am J Clin Nutr 2009;89:1173-1179

9. Troschel AS, Troschel FM, Best TD, et al. Computed tomography-based body composition analysis and its role in lung cancer care. J Thorac Imaging 2020;35:91-100.

10. Bridge $\mathrm{CP}$, Rosenthal $\mathrm{M}$, Wright $\mathrm{B}$, et al. Fully-automated analysis of body composition from $\mathrm{CT}$ in cancer patients using convolutional neural Networks. In: Stoyanov D, Taylor Z, Sarikaya D, et al, eds. OR 20 Context-Aware Operating Theaters, Computer Assisted Robotic Endoscopy, Clinical Image-Based Procedures, and Skin Image Analysis. New York, NY: Springer; 2018:204-213. 
11. Burns JE, Yao J, Chalhoub D, et al. A machine learning algorithm to estimate sarcopenia on abdominal CT. Acad Radiol 2020;27:311-320.

12. Magudia K, Bridge CP, Bay CP, et al. Population-scale CT-based body composition analysis of a large outpatient population using deep learning to derive age-, sex-, and race-specific reference curves [published online November 24, 2020]. Radiology, doi: 10.1148/ radiol.2020201640

13. Latenstein AEJ, Dijksterhuis WPM, Mackay TM, et al. Cachexia, dietetic consultation, and survival in patients with pancreatic and periampullary cancer: a multicenter cohort study. Cancer Med 2020;9:9385-9395.

14. Lorton CM, Griffin O, Higgins K, et al. Late referral of cancer patients with malnutrition to dietitians: a prospective study of clinical practice. Support Care Cancer 2020;28:2351-2360.

15. Millar C, Reid J, Porter S. Healthcare professionals' response to cachexia in advanced cancer: a qualitative study. Oncol Nurs Forum 2013;40: E393-402.
16. Vagnildhaug OM, Balstad TR, Almberg SS, et al. A cross-sectional study examining the prevalence of cachexia and areas of unmet need in patients with cancer. Support Care Cancer 2018;26:1871-1880.

17. Anandavadivelan P, Brismar TB, Nilsson M, et al. Sarcopenic obesity: a probable risk factor for dose limiting toxicity during neo-adjuvant chemotherapy in oesophageal cancer patients. Clin Nutr 2016;35:724-730.

18. Martin L, Birdsell L, Macdonald N, et al. Cancer cachexia in the age of obesity: skeletal muscle depletion is a powerful prognostic factor, independent of body mass index. J Clin Oncol 2013;31:1539-1547.

19. Food and Nutrition Board; Health and Medicine Division; National Academies of Sciences, Engineering, and Medicine. Examining Access to Nutrition Care in Outpatient Cancer Centers: Proceedings of a Workshop. Washington DC: National Academies Press; 2016.

20. Trujillo EB, Claghorn K, Dixon SW, et al. Inadequate nutrition coverage in outpatient cancer centers: results of a national survey [published online November 22, 2019]. J Oncol, doi: 10.1155/2019/7462940 